Etnográfica

Revista do Centro em Rede de Investigação em

Antropologia

vol. 25 (3) | 2021

Vol. 25 (3)

\title{
La escena nostálgica neobakala: transformación y ritualización musical de la cultura juvenil bakala
}

The neobakala nostalgic scene: musical transformation and ritualization of Bakala youth culture

\section{Eduardo Leste Moyano}

\section{(2) OpenEdition}

\section{Journals}

Edición electrónica

URL: https://journals.openedition.org/etnografica/10143

DOI: $10.4000 /$ etnografica. 10143

ISSN: 2182-2891

\section{Editor}

Centro em Rede de Investigação em Antropologia

\section{Edición impresa}

Fecha de publicación: 1 octubre 2021

Paginación: 707-728

ISSN: 0873-6561

\section{Referencia electrónica}

Eduardo Leste Moyano, «La escena nostálgica neobakala: transformación y ritualización musical de la cultura juvenil bakala», Etnográfica [En línea], vol. 25 (3) | 2021, Publicado el 29 octubre 2021, consultado el 09 enero 2022. URL: http://journals.openedition.org/etnografica/10143 ; DOI: https:// doi.org/10.4000/etnografica.10143

\section{(c) (i) (8)}

Etnográfica is licensed under a Creative Commons Attribution-NonCommercial 4.0 International License. 


\section{La escena nostálgica neobakala: transformación y ritualización musical de la cultura juvenil bakala}

\section{Eduardo Leste Moyano}

Los bakalas fueron una cultura juvenil que apareció en algunos lugares de España, entre ellos Madrid. Después de desaparecer, esta cultura ha sido reformulada de forma nostálgica por los neobakalas madrileños. Este artículo explora, a partir de datos etnográficos y entrevistas en profundidad, la forma en que estos neobakalas han transformado aquella cultura juvenil en una escena musical que ritualiza algunos de los elementos que la compusieron, especialmente sus músicas, produciendo un género musical. Además, este artículo constituye un esfuerzo por matizar ciertas formas de abordar la participación de personas adultas en escenas musicales, cuestionando la aplicabilidad del concepto de "ageing" (Bennet y Hodkinson 2012; Bennet 2013) y de "memoria cultural" (Bennet y Rogers 2016) en este caso de estudio. Por último, el caso de los neobakalas nos llevará a problematizar la forma en que St John $(1999,2011,2014)$ analiza la ritualidad de las escenas relacionadas con la música electrónica.

PALABRAS CLAVE: culturas juveniles, escenas musicales, nostalgia, ritualidad

The neobakala nostalgic scene: musical transformation and ritualization of bakala youth culture - The bakalas were a youth culture that showed up in some Spanish cities, Madrid among them. After disappearing, this culture has been recreated by neobakalas. This article, based on ethnography and in-depth interviews, explores how neobakalas from Madrid have transformed the bakala culture into a music scene that ritualizes some of the features of the bakalas, especially its songs, giving birth to a music genre. Furthermore, the case of bakalas and neobakalas will allow us to question the way Bennet (2013) and Bennet and Hodkinson (2012) have approached music scenes formed by adults. This work therefore interrogates the applicability of the concepts of ageing cultures (Bennet and Hodkinson 2012; Bennet, 2013) and cultural memory (Bennet and Rogers 2016) for the neobakala scene. The case of neobakalas will also allow us to problematize how St John (1999, $2011,2014)$ analyses the ritual practices of the music scenes related to electronic music.

KEYWORDS: youth cultures, music scenes, nostalgia, rituality

LESTE MOYANO, Eduardo (eduleste@gmail.com) - investigador independiente, España 


\section{INTRODUCCIÓN}

La nostalgia no es un fenómeno nuevo dentro de la industria cultural (Reynolds 2011 ). Sin embargo, su expansión en el ámbito de ciertas escenas musicales sí que lo es. En este sentido, desde hace unos años sucede que adultos vinculados al rock o al punk (Guerra y Pereira 2019) están reformulando su propio pasado nostálgicamente, dando lugar a profundas transformaciones en los usos y significados de estas producciones culturales, que surgieron en la segunda mitad del siglo XX. Dentro de esta dinámica nostálgica se encuentran los neobakalas madrileños, adultos que en su día fueron bakalas que, junto a otras personas más jóvenes, intentan reconstruir nostálgicamente las prácticas musicales del bakalao, ${ }^{1}$ un fenómeno juvenil vinculado a la música electrónica de baile que se dio en España de forma prácticamente paralela al del acid house británico en los años 80 pero que, en el caso madrileño, se extendió hasta bien entrados los 90 .

Este tipo de fenómenos musicales protagonizados por adultos han sido abordados por autores como Bennet and Hodkinson (2012), Bennet (2013) y Bennet and Rogers (2016), que han propuesto hablar de escenas musicales, dentro de las cuales aplicar un individualismo metodológico y tener en cuenta procesos de "ageing" y memoria cultural. Esta propuesta resulta útil para entender escenas en las que se pueda trazar una continuidad temporal o en las que los individuos que las componen puedan ser considerados como sujetos relativamente aislados, pero resulta problemático para otras. Es el caso de estos neobakalas madrileños, adultos insertos en dinámicas sociales compartidas que no han envejecido "dentro de", sino que recrean nostálgicamente las prácticas musicales de una cultura juvenil que desapareció, transformándola en una escena musical. Esta transformación supone, sobre todo, la ritualización de ciertos elementos del pasado, especialmente de las músicas que compusieron el bakalao, que en el pasado tuvieron un sentido rupturista, vanguardista, ecléctico e innovador pero que en el presente adquieren un sentido conservador, retrospectivo, purista y estático. Esta ritualización, articulada desde los conceptos de rituoide y ritual (Turner 1982, 1988 [1969]), me permitirá ilustrar las profundas diferencias entre bakalas y neobakalas y, además, cuestionar ciertos usos

I Esta etiqueta surge en la tienda valenciana de discos Zic Zac. Cuando en aquella época llegaban nuevos discos algunos jóvenes se referían a ellos como "bacalao de Bilbao" (Oleaque 2004). Bilbao es otra ciudad costera española donde uno de los platos "típicos" es el bacalao con lo que estos jóvenes establecieron un paralelismo entre el bacalao - como mercancía y plato - y la música que llegaba a la tienda. El término cuajó entre los jóvenes y terminó siendo usado por los medios para referirse ya no solo a la música sino a todo el fenómeno, que finalmente quedó referenciado como "bakalao". Este fenómeno se extendió durante más de diez años en los que, como veremos, se produjeron cambios. En otros trabajos (Leste 2018) en los que me he centrado en el pasado, esos cambios me han hecho hablar de bacalao, bakalao y vakalao, para establecer etapas de producción. En este artículo, en el que me centro en el presente, no entraré en tantos detalles y usaré la referencia genérica de bakalao (y bakalas) para referirme al pasado. 
que se han hecho de estos mismos conceptos, especialmente cuando hablamos de música electrónica (St John 1999, 2011, 2014). Usos que han tendido a ignorar la importancia del contexto cuando hablamos de rituales (algo que, como veremos, es extensible al concepto de escena de Bennet). Por último, esta transformación o ritualización del pasado, que tiende a solidificar ciertos aspectos del mismo por medio de una serie de reglas, me llevará a plantear la aplicabilidad de otro concepto que se articula con los de escena y ritualidad, como es el de género musical (Fabbri 1981; Frith 1998).

A modo de síntesis, este artículo analiza las transformaciones que los neobakalas hacen de la cultura bakala, poniendo especial énfasis en sus músicas. Para ello, este trabajo constará de dos partes. En la primera articularé un marco teórico apropiado para abordar tanto la nostalgia de la escena neobakala como la memoria de los bakalas, centrándome más en lo primero que en lo segundo, pues lo que principalmente me interesa tratar en este artículo es el presente y no el pasado. En la segunda parte presentaré la cultura bakala, reconstruida a partir de entrevistas en profundidad, y la escena neobakala, abordada desde una etnografía expandida (Domínguez Figaredo 2012). ${ }^{2}$ De esta forma, podremos observar cómo los neobakalas transforman las producciones culturales de los bakalas.

\section{“AGEING” Y ESCENAS MUSICALES}

Como ya he señalado, los neobakalas son adultos que participan de la música popular. Para abordar este tipo de fenómenos, es decir, para abordar la presencia y persistencia de adultos dentro de este campo, Bennet and Hodkinson (2012) propone un concepto de escena musical que supere la asociación música-juventud y en el que quepan las particulares (e infinitas) formas de participar que tienen los adultos dentro de las mismas. Para recoger esta pluralidad participatoria de los adultos, Bennet and Hodkinson (2012), Bennet (2013) y Bennet and Rogers (2016) recurre a un individualismo metodológico (que elude las relaciones de poder en pro de la recolección de la subjetividad de cada participante) y sugiere una doble vía de interpretación conceptual. De esta forma, el sociólogo, por un lado, propone el concepto de "ageing" para hacer referencia al proceso de envejecimiento que se ha producido entre quienes un día abrazaron una filiación musical y nunca la abandonaron (Bennet and Hodkinson (2012); Bennet (2013), y, por otro, propone reinterpretar las escenas musicales como fenómenos "trans-temporales" (Bennet y Rogers 2016: 1). En este sentido, Bennet y Rogers señalan que los estudios sobre escenas musicales han obviado que la naturaleza de estas es, fundamentalmente, mnemónica (Bennet and Rogers 2016: 34), es decir, que dentro de ellas el pasado es un componente básico. Y es que para Bennet y Rogers (2016) la música (bien sea esta en forma 
de concierto tributo o de reedición de un disco) o el cine (documentales sobre el pasado del punk, por ejemplo) constituyen elementos básicos de las escenas y, además, medios a través de los cuales los distintos sujetos se relacionan con su pasado para situarse en el contexto presente, construyendo de esta forma su "memoria cultural". Individualismo metodológico, ausencia de relaciones de poder, pluralidad, envejecimiento, contextualización, mediaciones y memoria cultural constituyen, por tanto, los elementos básicos de una propuesta de escena musical que, si bien puede resultar útil para cuestionar imágenes preconcebidas de las escenas musicales (la relación música-jóvenes), resulta problemática en otros aspectos.

Algunos de estos aspectos ya han sido señalados por otros autores (Mendívily Spencer 2016) que han criticado la ausencia de relaciones de poder dentro de las escenas tal como las conciben Bennet (1999), Bennet and Hodkinson (2012), y Bennet and Peterson (2004). Ahora bien, de las escenas musicales, según las interpreta Bennet, no sólo están ausentes estas relaciones de poder sino también el contexto socioeconómico. De esta forma, resulta sorprendente que uno de los factores contextuales más importantes de estos años, como es la crisis de 2008 y sus efectos, no aparezca por ningún lado en sus trabajos de 2012, 2013 o 2016, en los que se ocupa de la persistencia de adultos en las escenas musicales. En cierto modo, es como si los informantes de Bennet vivieran como individuos aislados en un entorno de pleno empleo y al margen de cualquier conflicto político o social. ${ }^{3}$ Este contexto, que puede ser cierto para los casos que maneja Bennet, es, sin embargo, muy poco representativo del contexto en que se desenvuelven y actúan los neobakalas. Y es que, en España, en 2013 o 2016, adultos (y jóvenes) tenían (y tienen) que lidiar con graves problemas socioeconómicos derivados de la aplicación de los dogmas neoliberales impuestos para salir de la crisis de 2008.

Ahora bien, que el concepto escena musical de Bennet sea mejorable en estos aspectos no quiere decir que el concepto de escena no sea útil o sea "impreciso", como sostiene Hesmondhalgh (2005), debido al exceso de fluidez o individualismo del mismo. En este sentido, el concepto de escena musical no tiene porqué ser impreciso, siempre y cuando no entendamos por el mismo la suma de las particularidades de los distintos individuos que la conforman, como hace Bennet and Hodkinson (2012), Bennet (2013) y Bennet and Rogers (2016), sino la síntesis de los elementos que comparten, aunque esto que comparten "sólo" sea la dinámica de la interacción de los participantes entre sí y/o la que se da entre estos y otras escenas musicales, como hacía Straw (1991). Dinámica que, en el caso de los neobakalas, viene definida por el conflicto y la lucha por la autenticidad (Stokes 1997; Martí 2014). A fin

3 Otros autores (García 2015), sí han recogido los conflictos generados por la globalización dentro de las escenas musicales. 
de cuentas, si los neobakalas ritualizan las músicas del bakalao, produciendo un género musical, es para ganar cohesión interna y poder relacionarse con otras escenas musicales en términos de superioridad.

\section{NOSTALGIA Y MEMORIA CULTURAL}

Estos no son, de todas formas, los únicos aspectos que resultan problemáticos en el trabajo de Bennet and Hodkinson (2012), Bennet (2013) y Bennet and Rogers (2016) cuando se ocupa de las personas adultas que participan de escenas musicales. Su forma de abordar la dimensión temporal de las mismas es otro de ellos. Y esto por cuatro motivos. Primero, porque el concepto de "ageing" presupone una continuidad temporal que no siempre se da, como veremos con el caso de la escena neobakala que no es resultado del envejecimiento de la cultura bakala. Segundo, porque Bennet, junto con Rogers (2016), para abordar el pasado, proponen un concepto de "memoria cultural" que nunca llegan a definir en su propio libro y cuya única referencia bibliográfica es una compilación de artículos llevada a cabo por Erll y Nünning (2008) que en realidad alberga enfoques muy diversos sobre el tema. ${ }^{4}$ De esta forma, cuando Bennet y Rogers (2016) hablan de memoria cultural no sabemos si se refieren a la memoria cultural definida por Assmann $(1995,2008)$ o a la crítica que hace Harth (2008), en ese mismo volumen, del propio concepto de memoria cultural. ${ }^{5}$ Del mismo modo, cuando Bennet y Rogers (2016) se quejan de la limitación conceptual y de la falta de trabajos que se centren en prácticas mnemónicas, no se entiende que no mencionen el trabajo de Olick (2008) en esa misma compilación, o los múltiples trabajos de, por ejemplo, Barry Schwartz (2000) y Schwartz y Schuman (2005). El tercer aspecto por el que resulta problemática la propuesta de Bennet y Rogers es porque interpretan toda relación con el pasado como memoria, confundiéndola en ocasiones con la nostalgia. En este punto, conviene tener en cuenta que la memoria y la nostalgia son dos cosas distintas que tienen campos teóricos propios. En este sentido, la memoria tiene más de pasado que se incrusta en el presente con potencialidad crítica y la nostalgia más de presente que se incrusta y embellece el pasado (Tomé 2016; Leste 2018). ${ }^{6}$ Siguiendo esta diferenciación parece razonable plantearse cuánto de pasado y memoria hay en conciertos tributo, reediciones de discos y

4 Erll y Nünning (2008), en la introducción de la obra, sólo se refiere al concepto de memoria cultural como un paraguas bajo el que agrupar distintos enfoques sobre la memoria. Para esto toma como referencia el concepto de cultura de la antropología geertziana que poco tiene que ver con la sociología de Bennet (Erll y Nünning 2008: 4).

5 Assmann (2008) acuña el concepto de memoria cultural, que opone al de memoria comunicativa para referirse a lo que para él es memoria de larga duración.

6 Como decía Ricœur (2001), la memoria es del pasado y a ella se accede por medio del recuerdo, la anamnesis o la reminiscencia, pero no por medio de la imaginación (algo más propio de la nostalgia). 
demás productos y prácticas que Bennet y Rogers interpretan como "memoria cultural" o "heritage marketing" (Bennet 2009). Y el cuarto aspecto que complica la forma en que Bennet y Rogers interpretan las escenas protagonizadas por adultos es que ambos autores, además de confundir en ocasiones memoria con nostalgia, descartan esta última como variable fundamental para el análisis. De esta forma, Bennet y Rogers (2016) señalan que la vinculación de estos sujetos con el pasado va más allá de la "mera nostalgia", algo que para ellos no es más que un pequeño acto empoderador en la vida cotidiana de los sujetos (Bennet 2013).

Este último punto es importante porque, al hacer esto, Bennet y Rogers no sólo dejan de lado aquellas escenas en las que, como la neobakala, el eje principal es la nostalgia y no la memoria, sino que no se adentran en un sentimiento que "responde a" y es "producido en" un contexto político, social, económico, tecnológico, mediático y cultural marcado por, precisamente, el contexto que en Bennet se desvanece: el del capitalismo globalizado (Appadurai 2001 [1996]; Hirai 2009; Tomé 2016). Un capitalismo globalizado que produce polarización social y constantes movimientos que dan lugar a procesos de desterritorialización y destemporalización que se traducen en continuas sensaciones de pérdida y alienación (Appadurai 2001 [1996]), especialmente en aquellas personas que, como muchos neobakalas, quedan al margen de las clases más favorecidas que habitan los nuevos centros de poder, las ciudades globales (Rodríguez y López 2010). La nostalgia es, por tanto, un sentimiento que va mucho más allá de lo individual y de pequeños actos empoderadores. La nostalgia es un sentimiento que emana de la pérdida y trata de paliarla, que se traduce en un relato que muchas veces presenta una cierta estructura, pero que luego es practicada dentro de la vida social, dando lugar a distintos discursos nostálgicos (Appadurai 2001 [1996]; Herzfeld 1997; Hirai 2009; Tomé 2016) practicados con fines estratégicos (Battaglia 1995; Orr 2017). Unos fines estratégicos que se enmarcan en relaciones de poder (Hirai 2009) que hacen que la nostalgia aparezca en ocasiones como un discurso o práctica de dominación (Rosaldo 1989) y, en otras, como uno de resistencia (Battaglia 1995). No obstante, bien sea empleada para un fin o para otro, o bien sea conservadora o progresista (Smith y Campbell 2017), la nostalgia no dejará de converger con un capitalismo que, acelerado por las tecnologías digitales, facilita su aparición al mismo tiempo que la explota. Sin entender la nostalgia de esta manera no podemos entender la escena neobakala ni las transformaciones que esta opera. Son las dificultades de este contexto las que llevan a los neobakalas a ritualizar un pasado imaginado.

\section{RITUOIDE, RITUAL Y GÉNERO MUSICAL}

En este sentido, conviene desplegar un nuevo andamiaje teórico que permita entender la ritualización que la escena neobakala hace de las músicas del baka- 
lao. Para esto, como mencionaba en la introducción, resultará útil el trabajo de Turner (1982, 1988 [1969]). Primero, porque aporta un modelo de análisis de la ritualidad que no se desvincula del contexto y, segundo, porque este modelo provee de dos tipos de prácticas (rituales y rituoides) que nos permitirán entender diferentes matices de las prácticas y experiencias de los bakalas y de los neobakalas. De todas formas, y ya que hablamos de música, que es el elemento que aglutina a la escena, también resultará útil el concepto de género musical (Fabbri 1981), que circula en paralelo al de ritualidad.

Para Turner (1982, 1988 [1969]), los rituales eran formas expresivas regidas por unas reglas determinadas, practicadas en estructuras sociales particulares (contextos) que podían ser alteradas por la propia acción del ritual, puesto que todo ritual implica una fase de liminalidad, ${ }^{7}$ y un sentimiento de communitas capaces de estimular la creatividad humana. ${ }^{8}$ Para Turner, este género, que era originario de las sociedades tribales, se descompuso en las sociedades industriales en otra serie de géneros que denominó rituoides, formas expresivas muy parecidas a los rituales, que contenían un componente liminoide más que liminal. Para Turner, una de las diferencias fundamentales entre ambos conceptos era que lo liminal está pautado por reglas concretas y ayuda al sistema a prevalecer, mientras que lo liminoide no responde a reglas tan estrictas y es fuente de subversión. Dicho de otra forma, lo ritual/liminal es fuente de respeto, mientras que lo rituoide/liminoide no. Para Turner lo ritual/liminal se podía observar en iglesias y lo rituoide/liminoide en la música popular o la literatura. Ambos conceptos me servirán para enmarcar las músicas de la cultura bakala (liminoide) y la neobakala (liminal) mostrando la utilidad de ambos conceptos aunque, a diferencia de lo que pensaba Turner, y como veremos más adelante, la música popular puede llegar a ser tan solemne y ritual como la iglesia. Y digo esto porque los neobakalas han creado un evento profundamente reglado.

Esta regulación será la que me lleve a hablar de las fiestas neobakalas como un género musical, entendido este como "un conjunto de eventos musicales cuyo curso es gobernado por unas reglas" (Fabbri 1981: 52) que, aun siendo "socialmente aceptadas", suelen albergar tensiones internas que hacen vivir esas mismas reglas en un precario equilibrio, que se mueve entre la necesidad de innovación y la de perpetuación y que, precisamente por estas tensiones, son de carácter dinámico (Frith 1998). Ritualidad y género musical, como vemos, no están tan lejos el uno del otro, ambos exigen reglas y están vinculados a tensiones sociales. Ahora bien, el concepto de género musical nos aporta,

7 Fase en la cual las estructuras sociales se desdibujan. Es lo que Turner (1988 [1969]) llamó “antiestructura".

8 Communitas fue definido por Turner como un "sentimiento de entendimiento mutuo en el nivel existencial" (Turner 1982: 48). 
además, una serie matices que la noción de ritual de Turner no tiene. Primero, porque detalla con más precisión el tipo de reglas que pueden aparecer en un ritual (formales y técnicas, semióticas, de comportamiento, ideológicas, jurídicas o comerciales) y, segundo, porque el concepto de género musical (Fabbri 1981), como las escenas, reconoce que estos también se definen por su relación con otros conjuntos de eventos. Escena, ritual y género musical, como vemos, pueden ser complementarios y en el caso de los neobakalas nos servirán para ilustrar tanto sus interacciones con otras escenas, sus ritualizaciones del pasado imaginado y sus propias tensiones internas.

En cualquier caso, y volviendo a Turner, sus conceptos han sido muy utilizados para explicar culturas o escenas relacionadas con la música electrónica, especialmente por St John (1999, 2011, 2014), que los ha empleado para tratar las culturas relacionadas con la música psytrance. Para St John (2014), las personas que participan de la cultura psytrance emplean una serie de tecnologías sensitivas para alcanzar estados liminales y, con esto, producir modos de vida alternativos. Según St John (2011), se trataría de una cultura "superliminal" protagonizada por personas que buscan "conexión" y "verdades" en el mundo moderno. Gracias a estas tecnologías espirituales o superliminales, los seguidores del psytrance "se sienten más vivos que nunca en estos entornos que en cualquier otro momento de la vida ordinaria" (St John 2014: 243).

Estas formas de utilizar a Turner, que se centran tanto en el concepto de liminalidad (y sus técnicas) para tratar de explicar la experiencia de la música electrónica y sus posibilidades creativas son muy interesantes en el nivel fenomenológico, pero sucede con ellas que se pierden en lo conceptual y dejan de lado lo contextual. De esta forma, cuando St John (2014) dice que sus informantes "se sienten más vivos que nunca en estos entornos que en cualquier otro de la vida ordinaria" (St John 2014: 243), no sabemos por qué, aunque podamos intuirlo. En este sentido, y desde el punto de vista de la ritualidad, parece que St John se centra demasiado en las cosas que suceden en la antiestructura, olvidándose de las que suceden en la estructura. Y con estructura no me refiero sólo a la estructura interna de una escena dada, aspecto que St John (1999) suele desarrollar de forma profusa, sino a aquella más amplia en la que la escena se desenvuelve, es decir, su contexto. De esta forma, cuando St John habla de conflictividad social, sólo lo hace de aquellos conflictos que se dan en el interior de la escena psytrance, pero no lo hace de los conflictos o dinámicas que se dan entre sus participantes, generalmente de clase media o alta (St John 2017), y el resto de la sociedad. Todo esto resulta especialmente chocante cuando fue el propio Turner (1982, 1988 [1969]) el que, cuando habló de culturas insertas en sociedades industriales, como las bandas de moteros o los hippies, nunca evitó mencionar la vinculación entre actividad laboral, clase social y producción cultural. 


\section{MEMORIA Y CULTURAS JUVENILES}

La escena noebakala, como ya he dicho, es una escena nostálgica, por lo que tiende a hablar más de las condiciones de su presente que de su pasado. Si queremos acercarnos a este y ver cómo opera la transformación que estas personas realizan del mismo necesitaremos un proceso de reconstrucción de memoria de las músicas que compusieron el bakalao para así poder comparar presente y pasado. Este proceso fue llevado a cabo por el autor de estas páginas por medio de 28 entrevistas en profundidad realizadas a personas que fueron bakalas. A partir de ellas fui extrayendo recuerdos hasta hilar memoria (Halbwachs 2004 [1925]; Díaz Viana 2005).

La memoria de los neobakalas, por otro lado, exigió su propio marco teórico, puesto que, en el pasado, estos sujetos no eran neobakalas, sino bakalas. Dicho de otra forma, en el pasado estos sujetos no constituían una escena musical sino una cultura juvenil (Feixa 1998). Y digo esto porque, tal y como se desprende de los recuerdos de los bakalas, en el pasado, el elemento que los aglutinaba no era sólo la música, sino que también era una estética particular. Además, en aquel tiempo no construían su identidad en contraposición a otras escenas musicales sino en contraposición a las culturas parentales, respecto a las cuales ocupaban una posición subalterna.

\section{LOS BAKALAS Y SUS MÚSICAS}

El bakalao nació en Madrid de la adaptación que una serie de jóvenes góticos y new romantics hicieron del bakalao valenciano a mediados de los años 80 (Leste 2017; Leste y Val Ripollés 2019), poco después de la Transición y de la Movida madrileña (Fouce 2006; Val Ripollés 2017). ${ }^{9}$ Desde un punto de vista musical, los primeros compases de este bakalao madrileño estuvieron marcados por la combinación de bandas punk y postpunk de origen anglosajón, como The Cramps o Cabaret Voltaire. Junto a ellas también sonó el avant garde de Liaisons Dangereuses y la incipiente EBM de Front 242, configurándose un estilo musical en el que las bases rítmicas se hicieron las protagonistas. A toda esta combinación se fue sumando, a partir de 1987, el incipiente new beat belga de formaciones como Public Relation, que consolidó el gusto por los bombos de cuatro por cuatro. Durante los años 80 , estas músicas circularon en forma de vinilos por las discotecas bakalas (Planta Baja o Specka) donde primaba una estética gótica de tipo "háztelo tú mismo" hecha a base de ropa negra, maquillaje y una cierta androginia (Leste y Val Ripollés 2020).

Hacia 1990 las músicas de los bakalas comenzaron a dejar atrás la EBM y el postpunk para dar cabida al hardcore (el sello Warp, por ejemplo), a la incipiente 
música trance y al primer gabber. De esta forma, por las nuevas estructuras productivas de los bakalas, es decir, por discotecas como X-kandalo o New World, comenzaron a circular estas músicas de procedencia británica, belga, alemana $\mathrm{u}$ holandesa, distribuidas por los sellos Zyx, R\&S, Bonzai o Dance Opera. Así llegaron, entre 1990 y 1992, las producciones de Joey Beltram, Torsten Fenslau, o Nasty Django, que tenían en común una aceleración y acentuación del bombo. Desde un punto de vista estético los bakalas de estos primeros años 90 también cambiaron con respecto a los 80. Abandonaron el negro e introdujeron un estilo menos andrógino y más basado en marcas de ropa como pantalones Levi's, zapatillas New Balance, camisetas Powell Peralta y cazadoras vaqueras también de Levi's. Dejaron con esto el estilo "háztelo tú mismo" de años anteriores.

Hacia 1993, no obstante, los bakalas volvieron a innovar musical y estéticamente. Musicalmente, estos años del bakalao estuvieron caracterizados por el abandono casi definitivo de la EBM y el postpunk y por el predominio de la música trance, hardtrance e incluso el Goa trance, músicas que, de nuevo, ganaban en velocidad e intensidad. Con esto, a las nuevas discotecas bakalas, como Over Drive, llegó la música de los belgas Jones \& Stephenson, que publicaron su trabajo más conocido en Bonzai ("The first rebirth"), o la de DJ Rope \& Marco Bailey, que hicieron lo propio en Dance Opera. Y también fue así como llegó a Madrid el Goa trance del sello Dragonfly, proveniente de la región india de Goa. Desde el punto de vista estético, la ropa se hizo más ceñida al cuerpo y empezaron a incorporar otros elementos, como las cazadoras bomber, los polos Fred Perry o los plumas Pedro Gómez. ${ }^{10}$ El resultado fue un tipo estético hipermasculinizado (García García 2010), que tomaba elementos de los skinheads neonazis.

Hasta aquí hemos visto una breve reconstrucción de la cultura bakala, prestando especial atención a sus músicas que, como hemos visto, lejos de tener un canon, estuvieron en constante innovación, ruptura y vanguardia. No obstante, hay que señalar que desde el punto de vista musical, muchos bakalas no tenían conocimientos musicales demasiado precisos. En aquel tiempo se referían a su música simplemente como bakalao y para describirla tenían que recurrir a onomatopeyas. Así lo atestigua la siguiente cita de una bakala, extraída de la etnografía realizada por Gamella y Álvarez Roldán (1999) a mediados de los 90:

“En todos sitios se escucha bakalao. Lo que pasa es que [...]. El de [...] Megadisk es mucho pu... O sea, muy... Muy rayante, está to'l el rato ñiaa, ñiaa." (Natalia, en Gamella y Álvarez Roldán 1999: 280) 
Esta falta de interés o conocimiento musical se puede corroborar mediante la memoria de uno de los bakalas entrevistados, que reconocía que entonces no sabían mucho de música:

"Entonces tampoco entendíamos mucho de música, a no ser que fueras el DJ. Nosotros íbamos a escuchar música y cogernos el pedo.” [César]

Los bakalas, por tanto, estaban relacionados con la música, pero no tenían un conocimiento preciso de la misma. Esta cultura, más que estar regida por un discurso musical definido, se basaba en una serie de estéticas y sobre todo en la práctica de "la fiesta", que consistía en ir a las discotecas a bailar y "pillarse el pedo", como decía César, en referencia al consumo de drogas como el éxtasis. Esta fiesta estaba caracterizada, según relatan, por una permanente sensación de "buen rollo", una sensación que relacionaban con bailar, ofrecer, invitar, conocer gente, tener empatía, abrazarse, dejar de lado la representación estricta de la masculinidad, perdonar:

"Lo que nos enamoró a todos es que un tío podía decirle a otro tío... 'Me cago en la puta, toma un cacho de pastilla' y se daban un abrazo y nadie iba a pensar nada malo ni ibas a ser más blando por eso y era fantástico porque todo el mundo estaba por la labor de que todo estuviera bien. Y te empujaban lo más mínimo al pasar y no te decía 'perdona'... No, te decían 'perdona' [enfatiza] Y te querían dar un cacho de pastilla porque te habían empujado un poco." [Marta]

Visto este testimonio, la música que escuchaban y los psicoactivos que tomaban, podemos decir que "la fiesta" tenía un fuerte componente subversivo, liminoide. En la fiesta, un evento muy poco reglado que podía durar días, en el que los DJ no eran conocidos (no ocupaban una situación de privilegio), buscaban alterar las reglas de la vida ordinaria. En la fiesta, la masculinidad se podía relajar y se podía compartir con extraños. Pero, además, en la fiesta, aquellos jóvenes tomaban sustancias prohibidas, escuchaban músicas eclécticas y traspasaban cualquier tipo de regulación horaria y de control parental al estar durante días fuera de casa. Fue en "la fiesta" donde estos jóvenes experimentaron esos sentimientos que tanto se parecen a la communitas. Fue en la fiesta donde, al ritmo de Nasty Django o DJ Rope \& Marco Bailey, estos jóvenes subvirtieron su posición subalterna en tanto que jóvenes.

\section{Bakalas en contexto}

Hasta aquí hemos visto algunos de los principales rasgos de los bakalas. Pero, para entender sus cambios y su desaparición es necesario explicar su contexto. Estas culturas juveniles se produjeron en la ciudad de Madrid, en un tiempo 
en el que España se asentaba en la democracia liberal y se insertaba en la economía capitalista globalizada después de la entrada en la CEE en 1986. Este proceso de integración en la globalización y en el proyecto europeo no solo se tradujo en la llegada de músicas belgas o alemanas a la ciudad de Madrid, como acabamos de ver, sino que también supuso la llegada de capitales extranjeros. Con esto, la crisis económica que atravesaba España a mediados de los 80 empezó a remitir. Entre 1986 y 1992 España, por tanto, consiguió dejar atrás la crisis y entrar en la modernidad económica, que también fue tecnológica y mediática. Fue en estos años que proliferaron las salas de cines y se liberalizó el sector televisivo, lo que supuso pasar de un solo canal estatal a cuatro, tres de ellos privados (Tele5, Antena3 y Canal+). Por estas pantallas empezaron a circular anuncios de marcas de ropa y películas plagadas de product placement. Fue en este contexto de expansión económica y mediática que los jóvenes bakalas dejaron de producir su estética con técnicas de "háztelo tú mismo" y pasaron a introducir marcas de ropa: Levi's, Nike... ${ }^{11}$

La situación económica, no obstante, empeoró en 1993, año en que ya he dicho que se produjeron cambios estilísticos y musicales en esta cultura juvenil. A principios de los años 90 la economía internacional experimentó una recesión que llegó a España a finales de 1992. Para los jóvenes esta crisis supuso que, de nuevo, crecieran sus tasas de paro. Entre los hombres de entre 20 y 24 años, por ejemplo, el paro pasó del 26\% en 1992 al 39\% en 1994. Y entre las mujeres lo hizo del 38\% en 1992 al 46\% en 1994 (Martín Serrano y Velarde Hermida 1996). La crisis fue tan fuerte que muchos jóvenes tuvieron que aportar lo poco que podían ganar a la economía doméstica (Martín Serrano y Velarde Hermida 1996). ¿Cómo pagar entonces fiestas que podían durar días y ropas de marca? La escasez de recursos hizo que muchos de los jóvenes bakalas empezaran a vender drogas para costearse las fiestas y que muchos otros jóvenes tuvieran que pedir las drogas en préstamo, lo que derivó en frecuentes impagos. Pero, además, algunos de los que querían ropa nueva y no se la podían pagar, empezaron a robarla a otros bakalas. El resultado fue que esta cultura derivó hacia prácticas de riesgo y la producción de jerarquías. Esto, a su vez, se reflejó en sus gustos estéticos: la música se hizo más rápida y agresiva y la estética también tendió hacia la hipermasculinidad (García García 2010), ciñéndose al cuerpo.

A finales de 1993 el fenómeno del bakalao que se daba en Madrid, pero también en Valencia, saltó a los medios en forma de pánico mediático (Gamella y Álvarez Roldán 1999). Este pánico moral, en plena crisis económica, fue utilizado por el gobierno socialista, que para dar una imagen de fortaleza aplicó

11 La tasa de paro juvenil, que en los 80 había llegado a rozar el 50\% entre algunos, se redujo hasta el 25\% en el caso de los hombres y el 35\% en el caso de las mujeres (Martín Serrano y Velarde Hermida 1996). 
las leyes antiterroristas al fenómeno del bakalao, especialmente en Valencia. La presión de las autoridades, el enrarecimiento del ambiente entre los bakalas y su propio envejecimiento (muchos de ellos recuerdan que dejaron de "salir de fiesta" a partir de 1996) hizo que esta cultura juvenil se extinguiera y quedara estigmatizada por el pánico mediático que la asoció exclusivamente a drogas $\mathrm{y}$ violencia.

\section{LA ESCENA NEOBAKALA Y SU MÚSICA: CONTEXTO DE LA ESCENA NEOBAKALA}

Entre 1996 y 2008 la economía española no paró de crecer. Fue el período del "milagro económico español" obrado por la burbuja inmobiliaria y la expansión del crédito. Durante este tiempo, los que fueron bakalas se dispersaron en la adultez. Muchos se casaron, compraron sus casas y tuvieron hijos en una situación de aparente estabilidad en la que Madrid se consolidó como ciudad global (Rodríguez 2007). Como tal, en la capital española avanzaron ciertos procesos comunes a este tipo de urbes, como son los de desterritorialización y los de desigualdad social. En este sentido, la sociedad madrileña empezó a polarizarse entre una nueva middle upper class (Rodríguez y López 2010) vinculada a los puestos cualificados de las grandes empresas transnacionales y toda una legión de trabajadores rasos cuyas condiciones laborales rara vez les llevaban a superar los 1000 euros mensuales. De un lado quedaban directivos, abogados de grandes despachos, algunos diseñadores o publicistas y, del otro, trabajadores de la hostelería, el comercio, los servicios personales o la construcción. En cualquier caso, el acceso al crédito, tanto hipotecario como al consumo, era tan fácil en este tiempo que las desigualdades quedaron eclipsadas (Rodríguez y López 2010).

Sin embargo, cuando el crédito internacional se cortó a finales de 2007 la burbuja inmobiliaria se pinchó y, con ella, se rompió el marco de estabilidad social. La falta de acceso a crédito colapsó una economía en la que el paro empezó a galopar, superando el 25\% en 2012.12 Para solucionar la situación se aplicaron los dogmas liberales, algo que acentuó la polarización, que había arrancado años atrás. Así es como en Madrid fueron apareciendo toda una masa de trabajadores, antaño llamada clase obrera, donde hoy caben los trabajadores pobres, el precariado (Standing 2013), las masas de desempleados o muchos trabajadores rasos. Y así es también como en Madrid se propagó el miedo, porque quien tenía un trabajo no quería perderlo y pasar a formar parte de lo que Hernández (2014) denominó el ejército de reserva, todas aquellas personas que pierden el trabajo y, para volver a trabajar, tienen que aceptar cualquier condición. En este contexto de crisis económica, de desestructuración social, 
de globalización y de desterritorialización, la nostalgia, ese sentimiento tan característico del capitalismo, y tan relacionado con las rupturas, encontró vía libre para su expansión. Una expansión que se produjo en un nuevo entorno mediático marcado por la popularización de una serie de nuevas tecnologías digitales que aceleran los procesos de desterritorialización y destemporalización (Leste 2018), dando lugar, entre otras cosas, a una importante transformación de las prácticas musicales urbanas (Fouce 2016).

\section{Interacciones y ritualización en la escena neobakala}

Entender este nuevo entorno mediático hiperdigital (Feixa, Fernández Planells y Figueras-Maz 2016) es clave para entender la génesis y formación de la escena neobakala, porque fue por medio de los grupos de Facebook que algunos de los que habían sido bakalas entraron el contacto. Fue en Facebook donde algunos ex bakalas decidieron crear grupos para reunirse con otros participantes de "la fiesta". Y fue en este nuevo contexto que esta reunión de ex bakalas se articuló con otras músicas, dando lugar a una escena. Estas otras músicas o escenas con las que dialogan los neobakalas son el reggaeton, que asocian muchas veces con migrantes latinos, la EDM, ${ }^{13}$ que identifican con los jóvenes, y lo que ellos denominan minimal-techno, ${ }^{14}$ personas que interpretan como excesivamente sofisticadas. Es en contraposición a estas escenas y sus músicas, y a los grupos sociales que las sustentan, que la escena neobakala organiza su identidad. De esta forma, la escena neobakala rechaza el reggaeton por su exceso de "sexualización"; la EDM por estar protagonizada por jóvenes que no tienen "cultura", porque sus DJ (Steve Aoki, por ejemplo) llevan sesiones pregrabadas y porque usan tecnologías digitales; y el minimal-techno, porque sus DJ no son "profesionales" (Ricardo Villalobos, por ejemplo), porque es una escena llena de personas que "van a exhibir cuerpo y no les gusta la electrónica de verdad". Para los participantes de la escena neobakala, en definitiva, esas otras escenas no son auténticas. Frente a ellos, la escena neobakala reformula la cultura bakala, y elabora un discurso musical nostálgico que les facilita pensarse en términos de superioridad. Según su forma de ver las cosas, las personas de otras escenas nunca sabrán lo que es escuchar y bailar música "de verdad", la del sello de R\&S, por ejemplo, en discotecas auténticas, como Over Drive, un lugar donde se tenían experiencias que los demás nunca podrán conocer. Es así como la nostalgia embellece el pasado y se convierte en distinción, en garantía "contra los recién llegados" (Appadurai 2001 [1996]: 76). Y es así como los neobakalas generan una de las principales transformaciones en relación a la cultura bakala, porque la música ahora cobra una relevancia que antes no tenía y porque la música del pasado deja de ser una herramienta subversiva 
que se usaba frente a las culturas parentales y se transforma en un discurso de dominación:

"Lo de antes sí que era música y no lo que les venden ahora a los niños" [Luis]

"Ya les gustaría a la juventud de esta generación haber vivido lo que nuestros ojos nuestros oídos y nuestros corazones han sentido." [Jesica]

Ahora bien, a esta reunión de ex bakalas que se dio en este nuevo contexto económico y tecnológico, no asistieron todos los que habían formado parte de aquella cultura juvenil, sino que lo hicieron de forma mayoritaria los perdedores de la globalización: los parados, los trabajadores rasos o el precariado. De esta forma, la mayoría de los informantes con los que pude tratar o estaban en paro o eran trabajadores poco cualificados que trabajaban en situaciones precarias. En este sentido, de los que trabajaban, la mayoría se dedicaba a actividades de comercio, hostelería, empleo doméstico, servicios personales, limpieza, mantenimiento de edificios o seguridad. También había varios trabajadores rasos: administrativos de grandes, medianas y pequeñas empresas cuyos sueldos pocas veces superaban los 1000 o 1400 euros. Esto no quiere decir que la totalidad de los que volvieron pertenezcan a estos estratos, sino que los que pertenecen a estos estratos son la mayoría. En general, son personas muy alejadas de los centros de poder. En este sentido, no pude encontrar a ningún participante de la escena neobakala que formara parte de cuadros directivos de grandes empresas. Esto no quiere decir que entre esos cuadros directivos no haya personas que hubieran sido bakalas. Las hay, e intenté entrevistarlas, pero todas rehusaron hablar de su pasado bakala. Se negaron, diciendo que o no entendían qué tenía aquello de interesante o porque se avergonzaban de ello. No hay que olvidar la imagen con la que quedaron los bakalas en el imaginario colectivo. Para ellos este pasado no suscitaba nostalgia, sino más bien remordimiento. En cualquier caso, los que sí entraron a formar parte de la reconstrucción del grupo han elaborado un discurso nostálgico que afirma su superioridad sobre las escenas musicales con las que se relacionan. Este discurso, además, no es sólo palabra, sino que es representado en las fiestas neobakalas.

Las fiestas neobakalas, a las cuales los participantes ya no asisten con una estética homogénea (como hacían los bakalas), tienen como principal objetivo "recordar" o "revivir" las experiencias del pasado y, para este fin, se estipulan unas reglas musicales que deben cumplirse. ${ }^{15}$ En primer lugar, la música de la fiesta ha de pertenecer exclusivamente a la citada en el apartado anterior

15 Revivir entrecomillo porque así se expresan las personas con las que trabajo. Pero como ya he dicho, los neobakalas más que recordar producen nostalgia. 
(EBM, new beat, hardcore, trance o gabber). No puede sonar de otros estilos o posterior en el tiempo (el límite lo marcan en 1996). En segundo lugar, se debe pinchar con vinilo. No se aceptan tecnologías digitales como las controladoras o programas del tipo Ableton, que automatizan las mezclas. En tercer lugar, la música ha de ir de menos a más. Cada tema tiene su momento. Estas son las tres reglas musicales básicas que deben asegurar uno de los principales objetivos de los neobakalas: el poder revivir la experiencia de la fiesta bakala, es decir, la communitas. ${ }^{16}$

Veamos un ejemplo para ilustrar estas reglas. En 2013, el promotor de la fiesta conmemorativa de la discoteca Over Drive invitó a DJ Abel. ${ }^{17}$ Este DJ es una persona muy respetada en la escena por su pasado en esa misma discoteca, pero, en su sesión de cierre de aquel año introdujo un disco de 1998 y otro de 2001. Este DJ fue fuertemente sancionado por muchos de los neobakalas que asistieron a la fiesta ese año:

"Fué patético... con [Fire Wire] se quedó parada la peña y encima mosqueados... fué un insulto... [...] por mi q lo veten para la proxima.” [Andrés]

DJ Abel no respetó las reglas y fue sancionado. Por el contrario, DJ Canito, otro artista que fue residente de Over Drive, cosechó mucho éxito en 2015. Veamos una comparación entre la sesión de DJ Abel de 2013 y la de Canito de 2016:

\section{Tabla 1}

Comparación de sesiones.

DJ Abel closing set 2013

- Jones \& Stephenson, “The First Rebirth”, hardtrance (1993)

- V-Tracks, "Subway 26", hardtrance (1994)

- Cosmic Gate, "Fire Wire", hardtrance (2001)

- Citizen X, "Running Out... (Of Time)" (MS-20 Mix), hardtrance (1998)

- Asphyx-, "XTC-Alarm” (Power Mix), hardtrance (1993)

- Rope \& Marco Bailey, “Spice”, hardtrance (1994)
DJ Canito closing set 2016

- Jones \& Stephenson, “The First Rebirth”, hardtrance (1993)

- Dreamland, "Mind Penetration", hardtrance (1993)

- Thunderball, "Bonzai Channel One", hardtrance (1993)

- RMB, "The Place To Be", hardtrance, gabber (2001)

- Play da Same Old Song, "Nasty Django", gabber (1992)

- Meditation, "Nacho Division”, hardtrance (1994)

Fuente: Elaboración propia. Para escuchar las referencias consultar $<$ https://bit.ly/2QFfyeH $>$

16 Como sugirió Connerton, para que un hechizo funcione éste debe ser siempre pronunciado de la misma manera (Connerton 1989: 48).

17 Se trata de una fiesta anual. 
Al contrario que DJ Abel, Canito sí que respetó las reglas musicales de las fiestas neobakalas. Los estilos eran acertados (trance y gabber) y las fechas de producción también (1993-1994). DJ Abel, sin embargo, puso dos discos de hardtrance que no podían haber sonado en Over Drive debido a su año de producción (uno de 1998 y otro de 2001). La música, por tanto, debe seguir unas reglas que garanticen o simulen una continuidad temporal (que, por otro lado, ha de garantizar la consecución de una continuidad social interna y externa, es decir, de la communitas). Vemos, por tanto, cómo la escena neobakala genera una nueva transformación con respecto a la cultura bakala. La música, que en el pasado tuvo un carácter innovador, era desconocida y empleada principalmente para bailar, adquiere ahora un sentido conservador, retrospectivo, purista y estático. La música de Nasty Django, Jones \& Stephenson o DJ Rope \& Marco Bailey, por tanto, ha invertido su significado. Ahora forma parte de un corpus musical estático y cerrado que ha de ser respetado y exige conocimientos precisos.

Esta regulación de la música de las fiestas neobakalas las constituye en un ritual o, dicho de otra forma, en un género musical (Fabbri 1981) al que los propios neobakalas se suelen referir como remember. No obstante, esta regulación establece una fuerte tensión dentro de la escena, que se da tanto entre el público neobakala como entre éste y los DJ/promotores, que son los encargados de la música que sonará. Esta tensión entre neobakalas se pudo ver, por ejemplo, en la fiesta Over Drive de 2019, momento en que la marca celebraba su $25 .^{\circ}$ aniversario. Para ese evento, el promotor decidió contar con algunos de los productores de música trance de los años 90, como los belgas Bonzai All Stars, cuyos discos (Jones \& Stephenson, por ejemplo) sonaron en las discotecas bakalas. En un momento de la sesión, los miembros de este sello decidieron mezclar un tema de trance con uno de Queen ("We will rock you") algo que desató la polémica. Algunos miembros de la escena se quejaron diciendo, como dijo Germán, que hacer eso era "como profanar una tumba". ${ }^{18}$ Otras personas de la comunidad, sin embargo, defendieron la actuación del DJ señalando que había sido un "momentazo" y que no se podía esperar escuchar siempre lo mismo:

"Pues a mí me flipó el mezclote con el 'We will rock you'. Hay que atreverse." [Teresa]

La tensión entre los miembros de la escena fue en ascenso y algunos de los neobakalas más mayores comenzaron a recriminar ciertas quejas, especialmente

$18 \mathrm{Al}$ hacer esto, Germán le da a la música un aire de solemnidad que difícilmente habría tenido en el pasado. Como decía Turner, lo liminal es fuente de respeto. 
si estas venían de personas que no habían sido bakalas. Se abrió entonces una pugna que uno de los participantes quiso zanjar de la siguiente manera:

"Una reflexión... todos los que [...] criticamos en este grupo... visitamos la sala Over Drive? Porque para poder comparar [...] tenemos que haber vivido esa época porque a lo mejor muchos hablan por hablar y ni siquiera fueron a esta sala y ni vivieron esa época." [Antonio]

Con este comentario Antonio estableció una jerarquía, basada en la experiencia, con la que quiso limitar la participación de otras personas. Se hacían así evidentes las tensiones y jerarquías internas de la escena, en la que muchas veces las personas más mayores de la escena, personas que fueron bakalas, son las que acumulan más prestigio.

En este sentido, los neobakalas han creado una serie de jerarquías internas inexistentes en la cultura bakala. En la escena neobakala no es lo mismo el prestigio que acumula un DJ que trabajó en la discoteca New World en 1992 que el de un asistente. Los DJ ahora son personas que acumulan un capital subcultural especial debido al papel que supuestamente desempeñaron en el pasado. De esta forma, estas personas son tratadas con un respeto especial en la vida cotidiana de la escena, que se da en los grupos de Facebook. Y, del mismo modo, los neobakalas que fueron bakalas parecen atesorar un prestigio especial con respecto a los neobakalas que, por edad, no lo vivieron. Estas son jerarquías que no existían dentro de la cultura bakala. Baste recordar, por ejemplo, que los DJ en el pasado eran muy poco conocidos.

En este sentido, la fiesta neobakala no parece tener tanto que ver con recordar el pasado como con la creación y puesta en juego de esta nueva estructura en el presente. De hecho, es esta nueva estructura la que nos permite entender el sentido de esta nueva fiesta. Y es que, en las fiestas neobakalas, el orden social de la misma es invertido y el DJ, por ejemplo, pese a su prestigio, debe plegarse a su público y darle lo que este exige. Y lo que este exige es volver a experimentar la communitas y con ella erradicar su propio orden interno, hacer como si todos fueran uno. Esto, en cierto sentido, podría ser calificado como liminoide, subversivo. Ahora bien, para llegar a esto, se han debido seguir previamente unas reglas y, por tanto, lo liminal se coloca como condición previa a lo liminoide. De esta forma, llegan a aquella paradoja señalada por Turner en la que "la experiencia de la communitas se convierte en la memoria de la communitas" (Turner 1982: 47). En cualquier caso, toda esta acción ritual y sus reglas han de ser entendidas dentro del contexto del orden social más amplio (externo) en el que viven los neobakalas. Si los neobakalas crean jerarquías, se aíslan en una discoteca y juegan con ellas es para liberarse, aunque sea momentáneamente, del malestar derivado de las desigualdades sociales, laborales, políticas, económicas y culturales. A fin de cuentas, la nostalgia no deja de ser un 
sentimiento que, además de ser producido en, es una respuesta a. Ahora bien, conviene no perder de vista que esta práctica que libera momentáneamente mediante la acción ritual es traducida en la vida ordinaria en una afirmación de las jerarquías y autenticidad que tratan de producir frente a las otras escenas con las que se relacionan, como el reggaeton. Frente a esos otros, frente a la globalización y la desigualdad que coloca a estas personas (generalmente de clase obrera) tan lejos del poder, los neobakalas han producido un género musical local dentro del cual, como dicen muchos neobakalas, es mejor el producto local (nacional) que el extranjero. Estos son los nuevos usos y significados de la música de los neobakalas.

\section{CONCLUSIONES}

Como hemos visto a lo largo del artículo, los neobakalas pueden ser entendidos como una escena musical siempre y cuando dentro de la escena musical se introduzcan en ellas variables como la del contexto socioeconómico, la de la clase social o las relaciones de poder. Sin estos aspectos difícilmente podríamos haber explicado la escena neobakala, que produce jerarquías internas y externas. Por otro lado, esta escena difícilmente puede ser entendida como una escena productora de "memoria cultural", como sugerían Bennet y Rogers (2016) sino de nostalgia. Los neobakalas, en este sentido, no pueden ser entendidos como una escena "transtemporal" en la que se produce un proceso lineal de envejecimiento bakala, sino que lo que se produce es una reformulación nostálgica de un pasado que es profundamente transformado, embellecido y olvidado.

En este sentido, hemos visto cómo los neobakalas transforman una cultura juvenil de tipo liminoide (subversiva, poco regulada) y dinámica en una escena musical de carácter liminal (usada para dominar y reglada) tendente al inmovilismo. Dicho de otra forma, a lo largo del texto hemos visto cómo los neobakalas producen un género musical que en el pasado no existía como tal. 


\section{BIBLIOGRAFÍA}

APPADURAI, Arjun, 2001 [1996], La Modernidad Desbordada: Dimensiones Culturales de la Globalización. Buenos Aires: FCE y Trilce.

ASSMANN, Jan, 1995, "Collective memory and cultural identity", New German Critique, 65: 125-133.

ASSMANN, Jan, 2008, "Communicative and cultural memory", in Astrid Erll y Ansgar Nünning (cords.), Cultural Memory Studies: An International and Interdisciplinary Handbook. Berlín: WdeG, 109-119.

BATTAGLIA, Debbora, 1995, "On practical nostalgia: self-prospecting among urban trobrianders", in Debbora Battaglia (coord.), Rhetorics of Self-Making. Berkeley, CA: University of California, 77-97.

BENNET, Andy, 1999, "Subcultures or neo-tribes? Rethinking the relationship between youth, style and musical taste", Sociology, 33 (3): 599-617.

BENNET, Andy, 2009, "Heritage rock: rock music, re-presentation and heritage discourse", Poetics, 37 (5-6): 474-89.

BENNET, Andy, 2013, Music, Style, and Aging: Growing Old Disgracefully? Filadelfia: Temple University.

BENNET, Andy, y Richard PETERSON, 2004, Music Scenes: Local, Translocal and Virtual. Nashville: Vanderbilt University Press.

BENNET, Andy, y Paul HODKINSON, 2012, Ageing and Youth Cultures: Music, Style and Identity. Londres: Berg.

BENNET, Andy, y Ian ROGERS, 2016, Popular Music Scenes and Cultural Memory. Londres: Macmillan.

CONNERTON, Paul, 1989, How Societies Remember. Cambridge: Cambridge University Press.

DÍAZ VIANA, Luis, 2005, "Los caminos de la memoria: oralidad y textualidad en la construcción social del tiempo”, Acta Poética, 26 (1-2): 181-217.

DOMÍNGUEZ FIGAREDO, Daniel, 2012, "Escenarios híbridos, narrativas transmedia, etnografía expandida”, Revista de Antropología Social, 21 : 197-215.

ERLL, Astrid, y Ansgar NÜNNING, 2008, Cultural Memory Studies: An International and Interdisciplinary Handbook. Berlín: WdeG.

FABBRI, Franco, 1981, "A theory of musical genres: two applications", in David Horn y Philip Tagg (coords.), Popular Music Perspectives. Göteborg y Exeter: International Association for the Study of Popular Music, 52-81.

FEIXA, Carles, 1998, De Jóvenes, Bandas y Tribus. Barcelona: Ariel.

FEIXA, Carles, Ariadna FERNÁNDEZ PLANELLS, y Mónica FIGUERAS-MAZ, 2016, "Generación hashtag: los movimientos juveniles en la era de la web social”, Revista Latinoamericana de Ciencias Sociales, Niñez y Juventud, 14: 107-120.

FOUCE, Héctor, 2006, El Futuro Ya Está Aquí. Madrid: Velecio.

FOUCE, Héctor, 2016, "The sound of the digital global city", Urbanities, 6 (1): 73-89.

FRITH, Simon, 1998, Performing Rites: On the Value of Popular Music. Cambridge, MA: Harvard University Press.

GAMElla, Juan Francisco, y Artuto ÁlVAREZ ROLDÁN, 1999, Las Rutas del Éxtasis: Drogas de Síntesis y Nuevas Culturas Juveniles. Barcelona: Ariel.

GARCÍA, Luis-Manuel, 2015, "At home, I'm a tourist: musical migration and affective citizenship in Berlin", Journal of Urban Cultural Studies, 2 (1-2): 121-134. 
GARCÍA GARCÍA, Antonio, 2010, "Exponiendo hombría: los circuitos de la hipermasculinidad en la configuración de prácticas sexistas entre varones jóvenes”, Revista de Estudios de Juventud, 89: 59-78.

GUERRA, Paula, y Thiago PEREIRA, 2019, “Nevermind... What?: memória, nostalgia e os tensionamentos possíveis entre o punk e o museu na exposição 'Nirvana: Taking Punk to the Masses (2017)'”, E-Compós, 23: 1-21.

HALBWACHS, Maurice, 2004 [1925], La Memoria Colectiva. Zaragoza: Prensas Universitarias de Zaragoza.

HARTH, Dietrich, 2008, "The invention of cultural memory", in Astrid Erll y Ansgar Nünning (coords.), Cultural Memory Studies: An International and Interdisciplinary Handbook. Berlín: WdeG, 85-96.

HERNÁNDEZ, Esteban, 2014, El Fin de la Clase Media. Madrid: Clave Intelectual.

HERZFELD, Michael, 1997, Cultural Intimacy: Social Poetics in the Nation-State. Londres: Routledge.

HESMONDHALGH, David, 2005, "Subcultures, scenes or tribes? None of the above", Journal of Youth Studies, 8 (1): 21-40.

HIRAI, Sinji, 2009, Economía Política de la Nostalgia: Un Estudio sobre la Transformación del Paisaje Urbano en la Migración Transnacional entre México y Estados Unidos. México, DF: Universidad Autónoma Metropolitana.

LESTE, Eduardo, 2017, “De Valencia a Madrid: bacalas madrileños: 1985-1989”, Anales del Instituto de Estudios Madrileños, 58: 559-583.

LESTE, Eduardo, 2018, Memoria y Nostalgia en la Industria Musical: el Caso de la Música Electrónica. Madrid: Universidad Complutense de Madrid, departamento de Periodismo y Nuevos Medios, tesis de doctorado.

LESTE, Eduardo, y Fernán VAL RIPOLLÉS, 2019, "Más allá del postpunk: góticos y new romantics entre Valencia y Madrid en los años ochenta", Resonancias, 23 (45): 215-239.

MARTÍ, Josep, 2014, "Music and alterity processes", Humanities, 3: 645-659.

MARTÍN SERRANO, Manuel, y Olivia VELARDE HERMIDA, 1996, Informe Juventud en España. Madrid: Instituto de la Juventud.

MENDÍVIL, Julio, y Christian SPENCER, 2016, "Introduction - Debating genre, class and identity: popular music and music scenes from the Latin American world", in Julio Mendívil y Christian Spencer (coords.), Made in Latin America: Studies in Popular Music. Nueva York: Routledge, 1-22.

OLEAQUE, Joan M., 2004, En Èxtasi. Barcelona: Aras Libres.

OLICK, Jeffrey, 2008, "From collective memory to the sociology of mnemonic practices and products”, in Astrid Erll y Ansgar Nünning (coords.), Cultural Memory Studies: An International and Interdisciplinary Handbook. Berlín: WdeG, 151-163.

ORR, Raymond, 2017, "The nostalgic native? The politics and terms of heritage and remembrance in two communities”, International Journal of Heritage Studies, 23 (7): 1-11.

REYNOLDS, Simon, 2011 , Retromania. Londres: Faber and Faber.

RICCEUR, Paul, 2001, La Memoria, la Historia y el Olvido. México, DF: FCE.

RODRÍGUEZ, Emmanuel, 2007, "Nuevos diagramas sociales: renta, explotación y segregación en el Madrid global”, Observatorio Metropolitano, Madrid: ¿La Suma de Todos? Globalización, Territorio, Desigualdad. Madrid: Traficantes de Sueños, 95-166.

RODRÍGUEZ, Emmanuel, y Isidro LÓPEZ, 2010, Fin de Ciclo: Financiarización, Territorio y Sociedad de Propietarios en la Onda Larga del Capitalismo Hispano (1959-2010). Madrid: Traficantes de Sueños. 
ROSALDO, Renato, 1989, Culture and Truth: The Remaking of Social Analysis. Boston: Beacon Press.

ST JOHN, Graham, 1999, Alternative Cultural Heterotopia: ConFest as Australia's Marginal Centre. Bundoora: La Trobe University, School of Sociology, Politics and Anthropology, tesis doctoral.

ST JOHN, Graham, 2011 , "Spiritual technologies and altering consciousness in contemporary counterculture”, in Michael Winkelman y Etzel Cardeña (coords.), Altering Consciousness: A Multidisciplinary Perspective Vol. I. Santa Barbara, Praeger Perspectives, 203-225.

ST JOHN, Graham, 2014, "Liminal being: electronic dance music cultures, ritualization and the case of psytrance", in Andy Bennet y Steve Waksman (coords.), The Sage Handbook of Popular Music. Londres: Sage, 243-260.

ST JOHN, Graham, 2017, "Charms war: dance camps and sound cars at burning man", in Graham St John (ed.), Weekend Societies. Nueva York: Bloomsbury, 219-244.

SCHWARTZ, Barry, 2000, Abraham Lincoln and the Forge of National Memory. Chicago: The University of Chicago Press.

SCHWARTZ, Barry, y Howard SCHUMAN, 2005, "History, commemoration, and belief: Abraham Lincoln in American memory, 1945-2001”, American Sociological Review, 70: 183-203.

STANDING, Guy, 2013, El Precariado: Una Nueva Clase Social. Barcelona: Pasado \& Presente.

SMITH, Laurajane, y Gary CAMPBELL, 2017, "Nostalgia for the future: memory, nostalgia and the politics of class", International Journal of Heritage Studies, 23 (7): 612-627.

STOKES, Martin, 1997, "Introduction: ethnicity, identity and music: the musical construction of place", in Martin Stokes (coord.), Identity and Music: The Musical Construction of Place. Oxford: Berg, 1-28.

STRAW, Will, 1991, "Systems of articulation, logics of change: communities and scenes", Cultural Studies, 5 (3): 368-388.

TOMÉ, Pedro, 2016, "Paisajes serranos en una economía política de los sentimientos", in Luis Díaz y Dámaso Vicente (coords.), El Patrimonio Cultural Inmaterial de Castilla y León: Propuestas para Un Atlas Etnográfico. Madrid: CSIC, 161-182.

TURNER, Victor, 1982, From Ritual to Theatre: The Human Seriousness of Play. Nueva York: PAJ.

TURNER, Victor, 1988 [1969], El Proceso Ritual: Estructura y Antiestructura. Madrid: Taurus. VAL RIPOLLÉS, Fernán del, 2017, Rockeros Insurgentes, Modernos Complacientes: Un Análisis Sociológico del Rock en la Transición (1975-1985). Madrid: SGAE. 